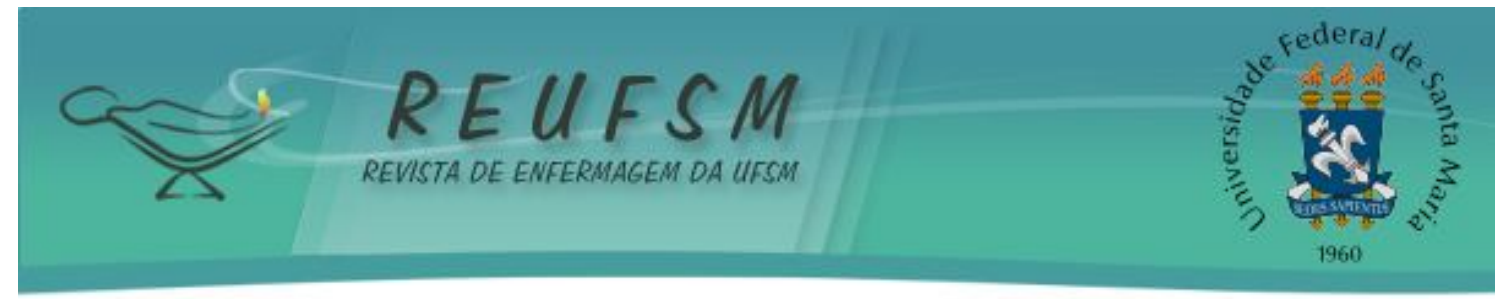

ARTIGO ORIGINAL

\title{
SINTOMAS DEPRESSIVOS E IDEAÇÃO SUICIDA EM ENFERMEIROS E MÉDICOS DA ASSISTÊNCIA HOSPITALAR
}

\author{
DEPRESSIVE SYMPT OMS AND SUICIDAL IDEATION IN NURSES AND PHYSICIANS CARE \\ HOSPIT AL
}

\section{LOS SÍNT OMAS DEPRESIVOS E IDEACIÓN SUICIDA EN ENFERMEROS Y MÉDICOS DE LA ATENCIÓN HOSPIT ALARIA}

\author{
Khivia Kiss Silva Barbosa ${ }^{1}$ \\ Kay Francis Leal Vieira ${ }^{2}$ \\ Estela Rodrigues Paiva Alves ${ }^{3}$ \\ Nereide Andrade Virgínio 4
}

RESUMO: Objetivo: investigar a presença de sintomas depressivos e ideação suicida entre enfermeiros e médicos da assistência hospitalar. Métodos: trata-se de uma pesquisa descritiva, com abordagem quantitativa, que contou com 100 sujeitos - 50 enfermeiros e 50 médicos, atuantes no contexto hospitalar no município de J oão Pessoa-PB, Brasil. Foram utilizados o Inventário de Depressão de Beck, a Escala de Ideação Suicida de Beck e um questionário sociodemográfico, com análise quantitativa. Resultados: mediante a análise dos dados, verificou-se que 30\% da amostra apresentou sintomatologia depressiva - 20\% enfermeiros e $10 \%$ médicos. A presença da ideação suicida foi verificada em $4 \%$ da amostra. Constatou-se, ainda, que todos eles também apresentaram depressão, o que evidencia uma forte relação entre os dois fenômenos. Conclusão: a prevalência de sintomas depressivos e a ideação suicida entre os profissionais de saúde apresentaram-se elevadas. Eles atuam contrariamente ao que se espera dessa população, tendo em vista sua atuação profissional.

Descritores: Enfermagem; Depressão; Suicídio; Saúde mental; Medicina.

ABST RACT: Objective: To investigate the presence of depressive symptoms and suicidal ideation among nurses and doctors in hospital care. Methods: This is a descriptive research with quantitative approach, which included 100 subjects - 50 nurses and 50 physicians working in hospitals in the city of João Pessoa, Brazil. We used the Beck Depression Inventory, Scale for Suicide Ideation, Beck and a sociodemographic questionnaire with quantitative analysis. Results: Based on the analysis of data showed that $30 \%$ of the sample had depressive symptoms - $20 \%$ and $10 \%$ nursing care. The presence of suicidal ideation was found in $4 \%$ sample. It was found also that all of them also had depression, which shows a strong relationship between the two phenomena. Conclusion: The prevalence of depressive symptoms and suicidal ideation among health professionals

\footnotetext{
${ }^{1}$ Enfermeira. Mestre em Enfermagem em Saúde Pública pela Universidade Federal da Paraíba. Docente da Universidade Federal de Campina Grande - UFCG e das Faculdades de Enfermagem e Medicina Nova Esperança (FACENE/FAMENE). E-mail: khiviakiss@yahoo.com.br

${ }^{2}$ Psicóloga. Doutora em Psicologia Social pela Universidade Federal da Paraíba. Docente da Faculdade de Enfermagem Nova Esperança (FACENE) e do Centro Universitário de J oão Pessoa (UNIPE). E-mail: kayvieira@yahoo.com.br

${ }^{3}$ Enfermeira. Mestre em Enfermagem em Promoção à Saúde pela Universidade de Pernambuco e Universidade Estadual da Paraíba (PAPGEn-UPE/ UEPB). Docente do Instituto de Educação Superior da Paraíba/IESP. E-mail: rodrigues.estela@gmail.com

${ }^{4}$ Enfermeira. Mestre em Enfermagem Fundamental pela Universidade Federal da Paraíba. Docente da Faculdade de Enfermagem Nova Esperança (FACENE). Enfermeira da Universidade Federal da Paraíba (Hospital Universitário Lauro Wanderley). E-mail: nereideav@uol.com.br
} 


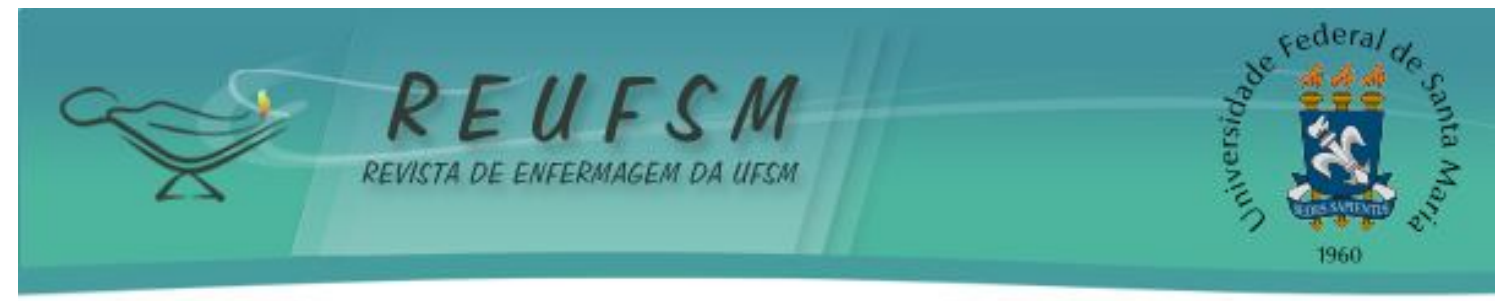

were el evated. They act contrary to what is expected of this population in view of their professional activities.

Descriptors: Nursing; Depression; Suicide; Mental health; Medicine.

RESUMEN: Objetivo: Investigar la presencia de síntomas depresivos e ideación suicida entre enfermeros y médicos y de la atención hospitalaria. Métodos: Se trata de un estudio descriptivo, con enfoque cuantitativo, que incluyó 100 sujetos siendo 50 enfermeros y 50 médicos que trabajan en el hospital de ciudad de João Pessoa/ PB/ Brasil. Fueron utilizados el Inventario de Depresión de Beck, Ia Escala de Ideación Suicida de Beck y una encuesta sociodemográfica con el análisis cuantitativo. Resultados: Los datos mostraron que el $30 \%$ de la muestra presentó síntomas depresivos, así siendo el $20 \%$ enfermeros y el $10 \%$ médicos. La presencia de la ideación suicida fue verificada en el $4 \%$ de la muestra. Se constató que todos ellos también tenían depresión, que muestra una fuerte relación entre los dos fenómenos. Conclusión: La prevalencia de síntomas depresivos y la ideación suicida entre los profesionales de la salud se elevaron. Esos síntomas actúan contra de lo que se espera de esta población en vista de su actividad profesional.

Descriptores: Enfermería; Depresión; Suicidio; Salud mental; Medicina.

\section{INTRODUÇÃO}

Os fenômenos da depressão e da ideação suicida encontram-se cada vez mais presentes na sociedade moderna e caracterizam-se, atualmente, como problemas de saúde pública. A depressão apresenta elevada incapacidade laborativa, alta morbidade e mortalidade. Remete ao desânimo e ao pessimismo, afeta a autoestima, diminui o interesse pelo mundo externo e a atividade sexual, causa insônia, falta de apetite, sentimentos autopunitivos e descrença em capacidades individuais. ${ }^{1}$

A atenção, a concentração e a memória podem ser prejudicadas devido à ruminação mental dos problemas e excessiva preocupação com problemas pessoais, dificuldades em manter contatos sociais e ideias suicidas. Tais sintomas confluem com as principais queixas dos pacientes na clínica atual que, são entre outras ${ }^{2}$, vida ausente de sentido, sentimento de vazio, de aniquilamento, perda de identidade e dificuldade de nomear as experiências e a solidão.

De acordo com a Organização Mundial da Saúde, para o ano 2020 ocorrerão, aproximadamente, um milhão e meio de suicídios em todo o mundo, ou seja, uma morte a cada vinte segundos. Assim, percebe-se que a depressão é uma doença mental incapacitante, pois leva ao surgimento de várias comorbidades e é um dos principais fatores de risco para o suicídio. Isso é particularmente preocupante ao se observar que grupos economicamente ativos, são mais vulneráveis a outros tipos de violências em nosso meio que podem levar ao suicídio.

A depressão poderá ultrapassar o número de pessoas afetadas por diabetes e doenças cardiovasculares. Na atualidade, é relacionada como a quarta causa mundial de deficiência e o segundo lugar na faixa etária compreendida entre 15 a 44 anos, podendo se tornar um problema crônico ou recorrente que impossibilite ao sujeito cuidar de si mesmo e de suas atividades diárias. ${ }^{4}$

Alguns profissionais são mais susceptíveis aos problemas de saúde mental, entre eles, o enfermeiro e o médico por interagirem, na maior parte do tempo, com pessoas que necessitam de ajuda. Inclusive, alguns fatores estressores, como clima de trabalho negativo, papéis ambíguos e a falta de clareza em relação às tarefas a serem executadas e às expectativas têm ef eitos adversos na saúde mental desses profissionais. ${ }^{5}$ 


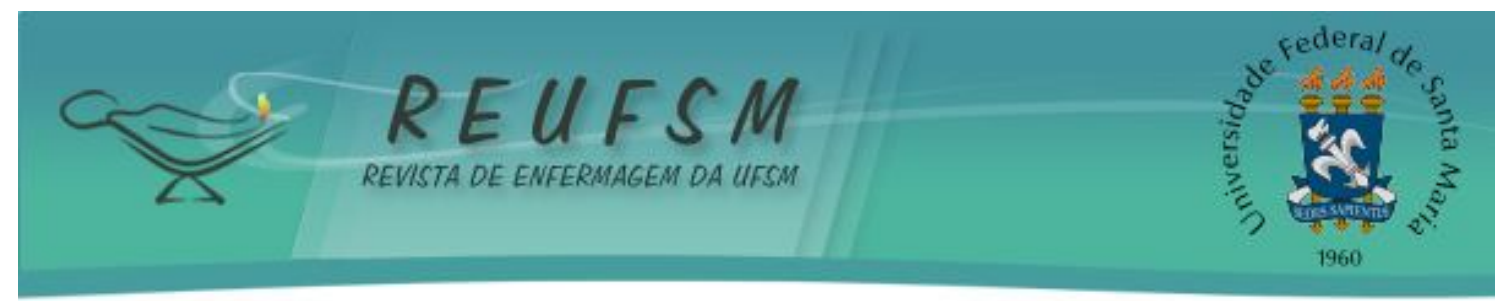

Nesse mesmo direcionamento ${ }^{6}$, excessivas jornadas de trabalho, acrescidas do estresse pela instabilidade do emprego, salários insatisfatórios e o fato de se deparar rotineiramente com a morte, com a dor e com o sofrimento também são fatores responsáveis por danos à saúde mental do profissional de saúde. Estudo ${ }^{7}$ constata que, em relação às horas de trabalho, o cumprimento de carga horária superior a 40 horas semanais estava presente em $41,7 \%$ dos participantes. Esses dados corroboram as pesquisas recentes que descrevem o excesso da carga horária de trabalho, somado a outras atividades cotidianas da equipe de enfermagem, como mediadores potenciais de desequilíbrios na saúde física e mental, além de desaj ustes na vida social e familiar desses profissionais.

A carga horária laboral excessiva e o próprio trabalho da enfermagem são tidos como alarmantes e podem ser importantes causas da deterioração da qualidade da assistência de enfermagem como gerador de ansiedade, sofrimento psíquico, estresse ocupacional, desgaste e fator gerador de insatisf ação profissional. ${ }^{8}$

Em relação à Medicina, especificamente, a literatura afirma que é uma profissão que requer muita dedicação por parte dos estudantes e que, quando formados, enfrentam, ao longo de suas carreiras, uma série de exigências profissionais tais como atuação altamente qualificada e formação continuada. No Brasil, pesquisas feitas com médicos têm encontrado indícios de sensação de fadiga e ideação suicida nesse grupo. Essas taxas são mais elevadas que as correspondentes à população em geral.. , 9-10

Não é possível exercitar a Enfermagem e a Medicina sem a consciência do bem e do mal, sem o desejo de servir, de ser útil ou sem o amor pelo ser humano. O profissional, algumas vezes, é obrigado a transformar o binômio profissional-paciente em um trinômio profissional-instituição-paciente e sofre pressões de interesses antagônicos, porquanto, de um lado, está a instituição, que exige produtividade, em termos quantitativos, ou seja, o máximo de atendimentos com o mínimo de despesas; de outro, o paciente, que requer atenção e qualidade. ${ }^{11}$ As características do trabalho em instituições de saúde possibilitam reconhecer agentes estressores importantes, detectados nos médicos que acompanham os estímulos emocionais relacionados ao adoecer. Muitas situações da prática médica se configuram como verdadeiros dilemas éticos e são altamente estressantes. A mesma situação é apresentada quando se observa a realidade na enfermagem.

Os sentimentos de culpa por fracasso de onipotência, impostos pelos limites de realidade, favorecem o surgimento de quadros depressivos e de ideação suicida, que são evidenciados por dados epidemiológicos da literatura, de incidência e prevalência, principalmente com os médicos, como mais elevado do que em toda a população. ${ }^{10}$ Por ideação suicida podemos entender como todo processo e causas de morte provocadas por uma ação da própria vítima com a percepção do seu resultado. ${ }^{12}$

Diante destes dados, foram elaboradas as seguintes questões norteadoras: quais os principais sintomas depressivos entre médicos e enfermeiros da assistência hospitalar do município de J oão Pessoa - PB? Existe a presença de ideação suicida entre enfermeiros e médicos da assistência hospitalar do município de J oão Pessoa - PB? Face ao exposto, a presente pesquisa objetivou investigar a presença de sintomas depressivos e ideação suicida entre enfermeiros e médicos da assistência hospitalar do município de João Pessoa - PB, considerando que esses profissionais, devido aos riscos iminentes do seu trabalho, são mais susceptíveis a prejuízos em sua saúde mental.

\section{MÉTODO}

Trata-se de estudo descritivo, com abordagem quantitativa, cuja amostra foi composta por 100 profissionais da área de saúde, de ambos os sexos - 50 enfermeiros e 50 médicos, atuantes no contexto hospitalar no município de J oão Pessoa-PB. 


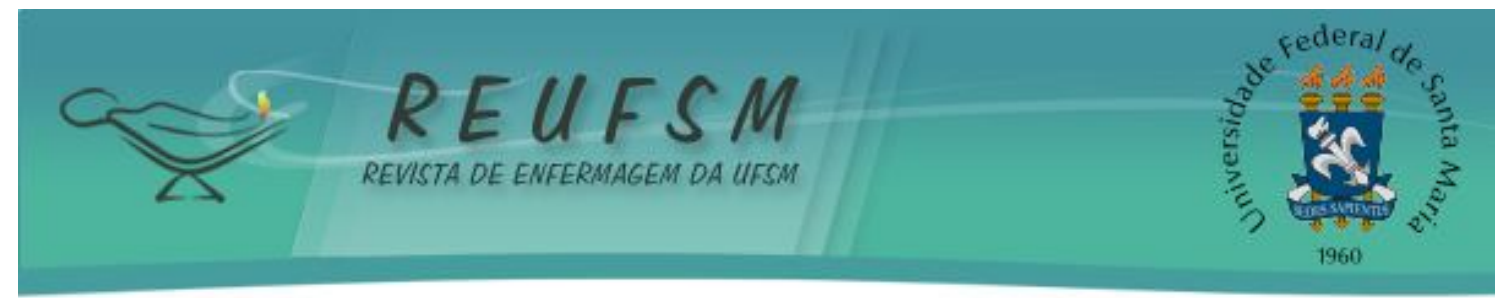

Os instrumentos empregados para a coleta dos dados foram o Inventário de Depressão de Beck (BDI), a Escala de Ideação Suicida de Beck (BSI) e um questionário sociodemográfico. O BDI é uma escala de autorrelato, de 21 itens, cada um com quatro alternativas, que subentendem graus crescentes de gravidade da depressão, com escores de 0 a $3^{13}$. É indicado para sujeitos de 17 a 80 anos, embora sejam citadas pesquisas desenvolvidas aquém e além desse período etário.

Já a BSI é um instrumento de autorrelato constituído por 21 itens, que foi estruturado de forma a permitir que os cinco primeiros itens fossem usados como triagem da ideação suicida. Assim, se a resposta do participante foi diferente de 0 no grupo de afirmações 4 - "indicando ausência de intenção ativa" - ou no grupo de número 5 "indicando evitação de morte, se confrontado com uma situação ameaçadora para a vida" - considera-se a existência da ideação suicida.

A coleta de dados foi realizada de fevereiro a abril de 2011 por dois alunos do Curso de Graduação em Enfermagem previamente treinados. Os instrumentos foram aplicados de forma individual, nos locais de trabalho dos participantes - três hospitais da rede pública.

O Protocolo do Projeto de Pesquisa foi aprovado pelo Comitê de Ética em Pesquisa (Protocolo 21/2010) com Seres Humanos em reunião dia 16/06/2010 com o Certificado de Apresentação para Apreciação Ética (CAAE) 0221.0.000. 351-10 das Faculdades de Enfermagem e Medicina Nova Esperança - FACENE/ FAMENE sob o que é referente a um proj eto de iniciação científica da referida instituição. Os autores não apresentam conflitos de interesse entre si.

Os participantes foram informados a respeito dos objetivos da pesquisa e da confiabilidade dos dados e do anonimato da sua colaboração. Em seguida, foi-Ihes solicitado que assinassem o Termo de Consentimento Livre e Esclarecido (TCLE), de acordo com as normas estabelecidas na Resolução do Conselho Nacional de Saúde (CNS) № 196/96, sobre pesquisas que envolvem seres humanos. ${ }^{14}$

Os dados apreendidos pelo Inventário de Depressão de Beck foram analisados seguindo-se os critérios de corte para os diferentes níveis de depressão ${ }^{13}$, conforme a classificação a seguir: 0-9, depressão mínima; 10-16, depressão leve; 17-29, depressão moderada; 30-63, depressão severa. Já a análise dos dados obtidos pela Escala de Ideação Suicida de Beck foi feita com a constatação de respostas diferentes de zero dadas pelo participante aos itens 4 e 5 . Os resultados apreendidos através desses instrumentos foram analisados com auxílio do programa estatístico SPSS, versão 18.0. Foram incluídos no estudo todos os enfermeiros e médicos que trabal havam na assistência hospitalar direta ao paciente, nos referidos hospitais, durante o período de coleta de dados, e que aceitaram participar do estudo mediante a assinatura do TCLE. Foram excluídos os enfermeiros e médicos que trabalhavam no hospital apenas na parte administrativa ou burocrática.

\section{RESULTADOS E DISCUSSÃO}

A preval ência de sintomas depressivos e a ideação suicida entre os profissionais de saúde apresentaram-se elevadas. Como já mencionado, a amostra total foi composta por 100 sujeitos (50 enfermeiros e 50 médicos), a maioria (62\%) do sexo feminino.

Segundo a faixa etária, constatou-se que $27 \%$ tinham idades entre 20 e 30 anos; $33 \%$ entre 31 e 40 anos; 24\% entre 41 e 50 anos; e 16\% idades superiores a 50 anos. De acordo com os resultados da pesquisa, o grupo que apresentou depressão era composto pelo sexo feminino e por profissionais enfermeiros e, apesar de os estudos refletirem um dado bem conhecido na epidemiologia da depressão ${ }^{15}$, deve-se considerar que a profissão é constituída por um público eminentemente feminino. 


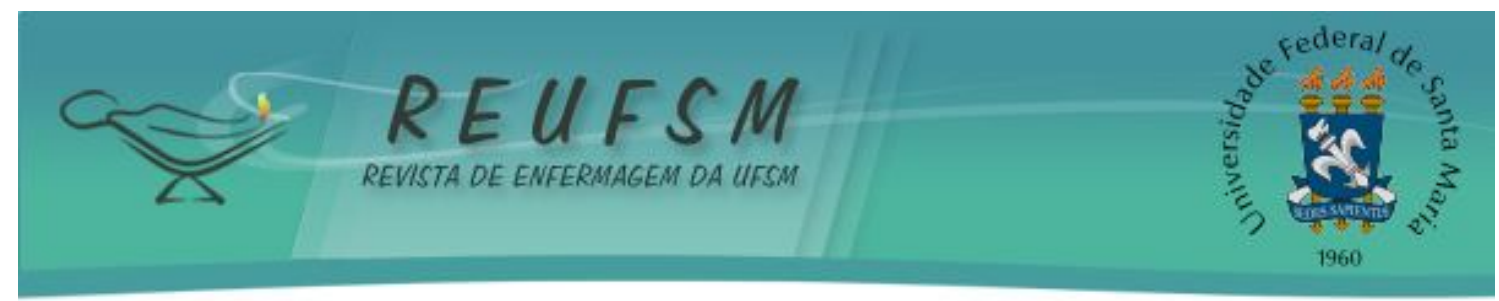

Tais dados reforçam os achados de alguns autores ${ }^{9}$, que constataram que $44 \%$ dos médicos apresentaram depressão ou ansiedade, e outras pesquisas ${ }^{11}$, identificaram um número de $86 \%$ de depressão nesses profissionais.

No que concerne ao estado civil dos participantes, a maioria (55\%) era casada, tinha filhos (66\%) e professava a religião católica $(57 \%)$. Em relação à atividade profissional, verificou-se que $51 \%$ trabalham em até dois locais; $40 \%$ em três ou quatro, e $9 \%$ tinham cinco ou mais empregos. Em relação ao turno de trabalho, a pesquisa mostrou que apenas $6 \%$ trabalhavam um turno; $34 \%$ dois turnos, e $60 \%$ três turnos.

As pressões no trabalho, a sobrecarga e os conflitos de interesses podem levar ao desequilíbrio mental, que se manifesta pela depressão. ${ }^{16} \mathrm{~A}$ exposição a ambientes de trabalho intensamente insalubres, como é o caso do hospital, também pode prejudicar a saúde devido às condições de trabalho precárias. Estima-se que a depressão será a segunda causa de incapacidade no mundo em $2020 .^{17}$

Esses dois fenômenos (depressão e ideação suicida) não são partes de uma única equação Iúcida, em que uma ocasiona a outra, mas entidades separadas que, com frequência, coexistem e se influenciam mutuamente. ${ }^{18}$

De acordo com a análise do BDI, os resultados expressam que $30 \%$ da amostra total apresentaram escores compatíveis com quadros depressivos - $19 \%$ com depressão leve, $7 \%$ moderada, e $4 \%$ severa.

Através do BSI, foi identificada a presença de ideação suicida em $4 \%$ da amostra total. É válido ressaltar que, em todos esses casos, o participante apresentou também episódio depressivo, conforme análise do instrumento anterior.

Alguns autores ${ }^{19}$ referem que a relação entre o transtorno mental e o suicídio já foi bem investigada. Diversos estudos em que se empregou a técnica de reconstrução diagnóstica conhecida (autópsia psicológica) têm indicado que o transtorno psiquiátrico é um dos maiores fatores de risco para o suicídio e que o comportamento suicida é bastante frequente entre a maioria dos grupos de pessoas com diagnósticos psiquiátricos. Porém, o transtorno mais preval ente entre vítimas de suicídio é o transtorno depressivo maior.

A partir dos dados apreendidos, através do questionário bio-sociodemográfico, foi possível traçar um perfil dos profissionais que apresentaram sintomas compatíveis com depressão, seja ela em nível leve, moderado ou severo.

Observou-se que, dos indivíduos com depressão ( $N=30), 66,67 \%$ eram enfermeiros, 0 que mostra que a prevalência de sintomatologia depressiva é mais acentuada entre os profissionais da enfermagem do que entre os médicos. Essa prevalência também foi constatada em indivíduos do sexo feminino $(80 \%)$, com idades entre 20 e 30 anos $(50 \%)$, com filhos $(63,33 \%)$ e de religião católica $(63,33 \%)$. No que concerne às atividades profissionais, a maioria tinha três ou quatro empregos $(56,67 \%)$ e trabalhava nos três turnos ( $80 \%)$.

Os dados desde estudo reforçam o pressuposto de que o acúmulo de atividades desenvolvidas em diferentes locais e a sobrecarga de horas de trabalho atuam como fatores desencadeantes da depressão. Nesse contexto, as condições apresentadas são totalmente adversas ao exercício satisfatório da profissão com dignidade e qualidade. ${ }^{20-21}$

Especificamente, o trabalho noturno, é um fator de risco para o desenvolvimento da depressão maior e profissionais de saúde com depressão podem não apresentar um desempenho adequado no trabalho ${ }^{21}$, o que afeta a assistência prestada por eles.

É preciso desenvolver novos estudos direcionados aos profissionais da área de Enfermagem, especialmente perante a constatação empírica do aumento do número de trabalhadores de enfermagem com depressão. ${ }^{22-23} \mathrm{Em}$ relação aos médicos, vale ressaltar que são "mestres" em negar as próprias doenças, apesar de conhecerem os sinais e os sintomas da depressão. ${ }^{24}$ 


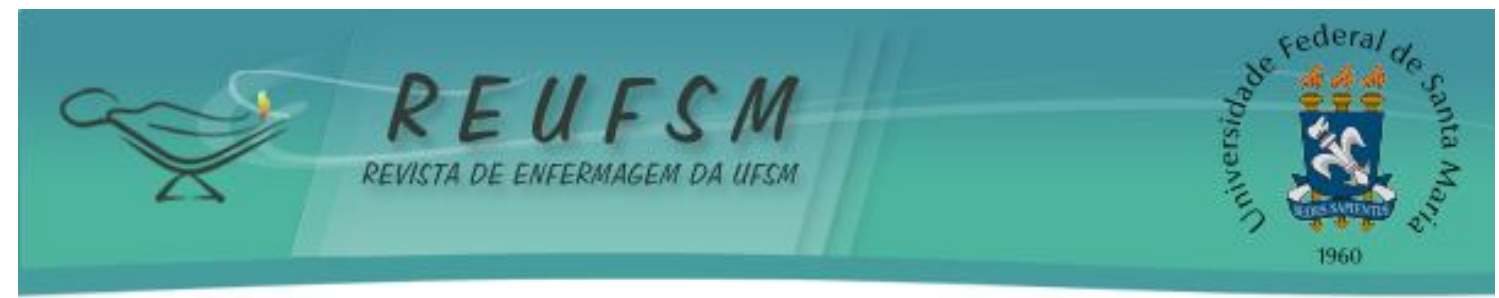

Vale salientar que não se pode furtar de reconhecer que a enfermagem cuida de pessoas e, nesse processo se lida com a subjetividade humana e com as múltiplas maneiras como tais pessoas vivem o sofrimento, a dor, as perdas e os ganhos, as alegrias e a tristeza. Para esta abordagem e entendimento necessários ao cuidado do outro, há que se praticar a solidariedade cujo sentido moral remete ao vinculo e à ajuda mutua ${ }^{25}$, tanto para a enfermagem quanto à medicina, que necessita também lidar com as adversidades e subjetividades humanas que permeiam a assistência hospitalar.

\section{CONSIDERAÇÕES FINAIS}

Os profissionais da área de Saúde, em sua dinâmica laboral, estão expostos às mais variadas formas de estímulos físicos e mentais, que os tornam mais susceptíveis a desenvolver sintomatologia depressiva e ideação suicida. Tal susceptibilidade decorre tanto do ambiente de trabalho quanto das tarefas desempenhadas porquanto lidam, cotidianamente, com doenças graves e com o risco eminente de morte do paciente.

0 alto índice de sintomas depressivos, assim como a presença de ideação suicida contrastam com o trabalho desempenhado por estes profissionais, de quem, geralmente, espera-se vir o cuidado.

Entretanto, enfermeiros e médicos, em alguns casos, devem receber apoio e acompanhamento de uma equipe multiprofissional com o objetivo de auxiliá-los a identificar seu sofrimento e, consequentemente, a desenvolver programas de prevenção e manutenção de sua saúde mental.

Os profissionais da área de Saúde precisam estar atentos para que a presença de transtornos mentais seja detectada e enfrentada antes que cause prejuízos ao seu desempenho profissional. Devem-se identificar os problemas psíquicos entre enfermeiros e médicos, com o fim de formular programas educacionais e estratégias clínicas para a orientação e o diagnóstico precoce, com o objetivo de prevenir a cronificação do transtorno depressivo, diminuir o risco do suicídio e 0 aumento da comorbidade com outros transtornos psiquiátricos.

Este estudo apresentou como limitações: a disposição por parte dos profissionais em responder os instrumentos de coleta, mesmo esta sendo previamente agendada, os mesmos demonstraram ansiedade em devolver rapidamente e se retirar do ambiente da pesquisa, alegando "falta de tempo"; bem como a escassez de dados de pesquisas recentes voltadas para este público, uma vez que a maioria os estudos se voltam para a população geral.

Acredita-se que tais fatos apontam para uma menor conscientização da real situação de saúde mental de enfermeiros e médicos que atuam na assistência hospitalar implicando risco de desenvolvimento de depressão e ideação suicida nessa população.

\section{REFERÊNCIAS}

1. Cambaúva LG, Silva Júnior MC. Depressão e neoliberalismo: constituição da saúde mental na atualidade. Psicol Ciênc Prof [internet]. 2005. [acesso em 2012 mar];25(4). Disponível em: http:/ / pepsic. bvsalud. org/ scielo. php?pid=S1414-

$98932005000400003 \&$ script $=$ sci_arttext.

2. Lowenkron AM. Sobre a clínica psicanalítica da atualidade: novos sintomas ou novas patologias? Rev Bras Psicanál. 2003 [acesso em 2012 mar]; 37(4). Disponível em: http:// www. rbp. org.br/ volumes. php?id_publicacao=10374.

3. WHO - World Health Organization. International Statistical Classification of Disears and Related Health Problems [internet]. 10 th Revision. Versão on-line 2003. [acesso em 2012 mar]. Disponível em: www. who.int/ classification/apps/ icd/ icd10online. 


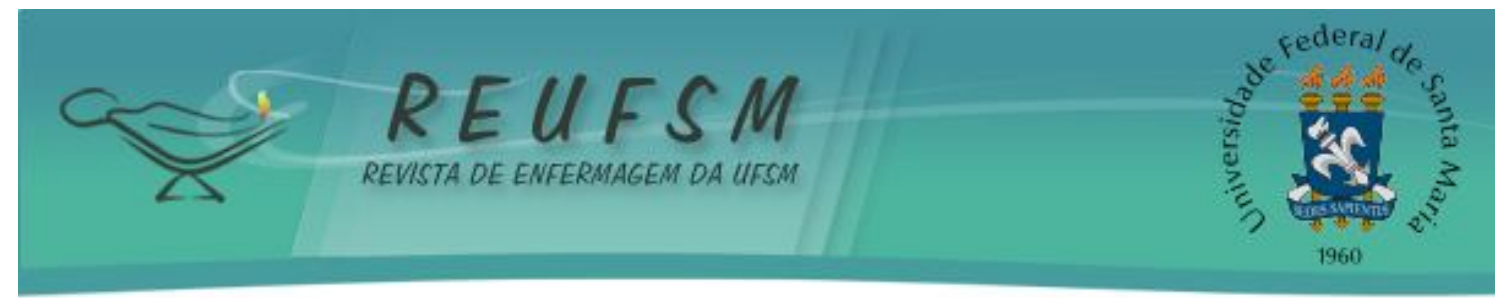

4. Monteiro KCC, LAGE AMV. Depressão: uma 'psicopatolologia' classificada nos manuais de psiquiatria. Psicol Ciênc Prof. 2007 [acesso em 2012 mar];27(1): 106-19. Disponível em: http:/ / pepsic. bvsalud.org/ scielo. php?pid =S1414-98932007000100009\&script=sci_arttext

5. Manetti MS, Marziale MHP. Fatores associados à depressão relacionada ao trabalho de enfermagem. Estud Psicol. 2007 [acesso em 2012 mar];12(1):79-85. Disponível em: http:/ / www. scielo.br/ scielo. php?script=sci_arttext\&pid=S1413-294X2007000100010

6. Oliveira GF, Barbosa GA, Souza LEC, Costa CLP, Araújo RCR, Gouveia VV. Satisfação com a vida entre profissionais da saúde: correlatos demográficos e laborais. Rev Bioét [internet]. 2009 [acesso em 2012 mar]; 17(2):319-34. Disponível em: http:/ / revistabioetica. cfm. org. br/index. php/ revista_bioetica/ article/ view/ 170/ 175

7. Lino, MM. Qualidade de vida e satisfação profissional de enfermeiras de Unidades de Terapia Intensiva [tese]. São Paulo (SP): Universidade de São Paulo, Escola de Enfermagem; 2004. 223 p.

8. Santos, LSC. Guirardello, EB. Demandas de atenção do enfermeiro no ambiente de trabalho. Rev Latinoam Enferm. 2007 [acesso em 2012 mar];15(1). Disponível em: http:/ / www. scielo.br/ scielo. php?pid=S0104$11692007000100005 \&$ script $=$ sci_arttext $\&$ tlng=pt

9. Marziale MHP. Enfermeiros apontam as inadequadas condições de trabalho como responsáveis pela deterioração da qualidade da assistência de enfermagem. Rev Latinoam Enferm. 2001[acesso em 2012 mar]; 9(3). Disponível em:

http:/ / www. scielo.br/ pdf/ rlae/ v9n3/ 11491. pdf.

10. Barbosa GA, Andrade EO, Carneiro MB, Gouveia VV. A saúde dos médicos no Brasil. Brasília: Conselho Federal de Medicina; 2007 [acesso em 2012 mar]. Disponível em: http:/ / www. portalmedico.org. br/ include/ asaudedosmedicosdobrasil. pdf.

11. Meleiro AMAS. Suicídio entre médicos e estudantes de medicina. Rev Assoc Méd Bras. 1998 [acesso em 2012 mar]; 44(2): 135-40. Disponível em:

http:/ / www. scielo.br/ pdf/ ramb/ v44n2/ 1993.pdf.

12. Durkheim E. O suicídio. 1aㅡ ed. Lisboa: Presença; 2003.

13. Cunha J A. Manual da versão em português das Escalas de Beck. São Paulo: Casa do Psicólogo; 2001.

14. Brasil. Ministério da Saúde. Resolução no 196, de 10 de outubro de 1996. Diretrizes e normas regulamentadoras de pesquisas envolvendo seres humanos. Brasília; 1996.

15. Capitão CG, Almeida FP. A incidência de depressão entre médicos que exercem suas atividades clínicas em ambulatórios. Psicol Hosp. 2006 [acesso em 2012 mar]; 4(2).

Disponível em: http:/ / pepsic. bvsalud. org/ scielo. php?pid=S1677-

$74092006000200005 \&$ script=sci_arttext.

16. Araya R, Rojas G, Fritsch R, Acuña J, Lewis G. Common mental disorders in Santiago, Chile. Br J Psychiat [internet]. 2001;[acesso em 2012 mar]; 178:228-33. Disponível em: http:/ / bj p. rcpsych. org/ content/ 178/ 3/ 228. short.

17. Baba V, Galaperin BL, Lituchy TR. Occupational mental health: a study of work-related depression among nurses in the Caribbean. Int J Nurs Stud [internet]. 1999 [acesso em 2012 mar];36: 163-9. Disponível em: http:/ / www. ncbi.nlm. nih. gov/ pubmed/ 10376226. 


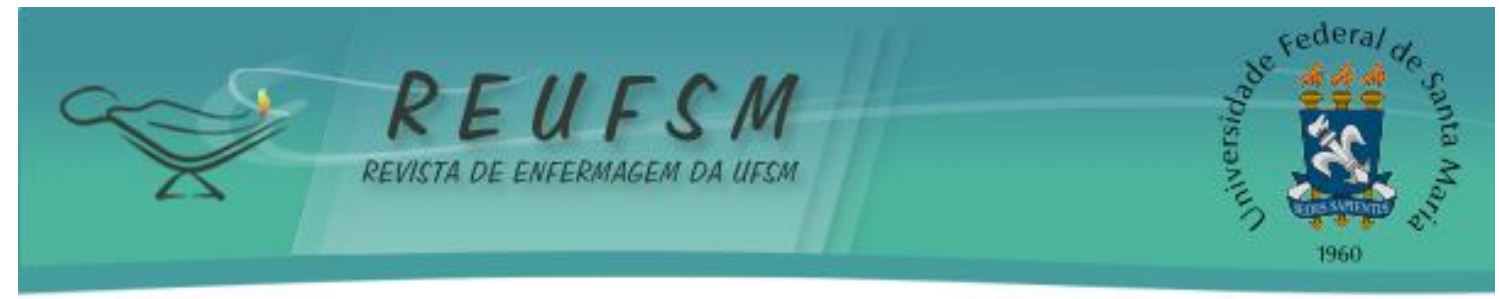

18. Muray CJ, Lopez AD. Global mortality, disability, and the contribution of risk factors: Global burden of disease study. Lancet [internet]. 1997 [acesso em 2012 mar];349: 1436-42. Disponível em: http:/ / www. thelancet. com/ journals/ lancet/ article/ PIIS01406736(96)07495-8/ abstract.

19. Solomon A. O demônio do meio dia. Rio de J aneiro: Objetiva; 2002.

20. Harris EC, Barraclough B. Suicide as an outcome for mental disorders: a meta-analysis. $\mathrm{Br} J$ Psychiatry [internet]. 1997 [acesso em 2012 mar];170:205-28. Disponível em: http:/ / bj p. rcpsych. org/ content/ 170/ 3/ 205. abstract.

21. Benevides AMTP. Burnout: quando o trabalho ameaça o bem-estar do trabalhador. São Paulo: Casa do Psicólogo; 2010.

22. Millan LR. Vocação médica: um estudo de gênero. São Paulo: Casa do Psicólogo; 2005.

23. Ruggiero J S. Correlates of fatigue in critical care nursing. Res Nurs Health. [internet] 2003 [acesso em 2012 mar];26: 434-44. Disponível em:

http:/ / onlinelibrary. wiley.com/ doi/ 10.1002/ nur. 10106/ abstract.

24. Meleiro AMAS, Almeida AM, Bartorelli B. O médico com depressão. In: Depressões em medicina interna e em outras condições médicas: depressões secundárias. São Paulo: Atheneu; 2001.

25. Ferreira MA. Sobre a solidariedade e a solicitude no cuidado de enfermagem. Rev Enferm UFSM [internet]. 2012 jan/abr;2(1). Disponível em:

http:/ / cascavel. ufsm. br/ revistas/ oj s2.2.2/ index. php/ reufsm/ article/ view/ 5150/ 3137

Data de recebimento: 19/07/ 2012

Data de aceite: 28/09/2012

Contato com autor responsável: Khivia Kiss Silva Barbosa

Endereço: Rua Enf. Ana M ${ }^{a}$ Barbosa de Almeida, 600/104 - Jardim Cidade Universitária J oão Pessoa, PB, Brasil.

CEP: $58052-270$

E-mail: khiviakiss@yahoo.com.br 